\title{
Emotions in ODR
}

Pre-publication version. Please quote: International Review of Law, Computers \& Technology, Volume 21, Number 2, July 2007, pp. 145-156.

\author{
Marta Poblet ${ }^{1}$ \\ Pompeu Casanovas ${ }^{2}$
}

\{marta.poblet; pompeu.casanovas\}@uab.cat

\begin{abstract}
For years, emotions have been widely considered in the areas of negotiation and conflict resolution. Standard methods of negotiation have dealt with the individuals' arousal and expression of a vast array of emotional states. If we consider ODR as a communicative process involving a group of individuals engaged in an interactive decision-making task, we will need to admit that emotions are an essential component in any online disputing process. This paper proposes a review of recent literature on emotions and ODR to discuss controversial issues such as the capacity of ODR techniques to deal with emotions and the advantages and disadvantages of computer-mediated communication versus face-to-face communication in terms of expressions of emotions.
\end{abstract}

\section{Introduction}

Research and theory about emotion has burgeoned in social and cognitive psychology, sociology, anthropology, economics, and neural sciences throughout the last two decades 1 . In the fields of decision-making and rationality, the integration of emotions in the models that predict human behavior paves the way to a new micro-foundation for the social sciences 2. As regards interactive decision-making, an extended research agenda has already been developed focusing on the interpersonal effects of emotions on negotiation processes. In this regard, there are many reasons to integrate the study of emotions within the conflict resolution, negotiation and, more recently, ODR research fields. First, negotiation and conflict resolution processes are social events necessarily involving interpersonal relations, and emotions may be considered as the "principal currency" of those relations 3. In Ekman's words, "emotions are brought into play most often by the actions of others, and, once aroused, emotions influence the course of interpersonal transactions" 4 . Second, the study of emotions can contribute to one of the

\footnotetext{
${ }^{1}$ Marta Poblet, Ph. D. in law (Stanford University), is a ICREA researcher at the Institute of Law and Technology of the Autonomous University of Barcelona (IDT-UAB) and lecturer of sociology of law at the UAB Department of Sociology.

${ }^{2}$ Pompeu Casanovas heads the Institute of Law and Technology of the Autonomous University of Barcelona (IDT-UAB) and is a professor of philosophy and sociology of law at the UAB Law School.
} 
most perennial and challenging issues that researchers on negotiation and decisionmaking, no matter their background, are face with: "to fill the gap between fixed action patterns and impeccable rationality" 5. It is well known that, for most approaches, the basic function of emotions is to mediate between individuals and their contexts 6 . In this view, emotions consist of flexible and adaptive responses that, while multifaceted, can be identified as specific behavioral components that may provide key elements in explaining and even predicting the outcome of negotiation processes. Finally, there is another practical reason to integrate emotions in the negotiation, conflict resolution and ODR agenda. Since interactive decision processes are often shaped by time-pressure, uncertainty, disruptions, changing conditions, attention paid to emotional issues may anticipate possible obstacles to conflict resolution and, as Lund has suggested, may also prevent mediator stress and burnout 7 .

Despite this significant amount of research, literature on emotions and the most common forms of dispute resolution (negotiation, mediation, arbitration and litigation) tend to focus primarily on traditional ADR techniques deployed in a face-to-face communication basis (F2FC). In contrast, much less attention has been given to emotions in computer-mediated-communication (CMC) and, more specifically, ODR. ${ }^{3}$

This paper proposes a review of recent literature relevant for the discussion about the expression of emotions in ODR. We start by introducing the most important approaches in emotions theory, and we continue by discussing the advantages and limits of ODR techniques in dealing with emotions. We suggest that the most usual criticisms to ODR methods can be counterweighed with recent research not so detrimental with the use of computer-mediated-communication. Finally, we conclude by identifying promising lines of research for the future of ODR.

\section{What is an emotion?}

The complexity of emotions, together with the difficulties to distinguish "emotions" from "moods", "temperaments", or "affective styles" might explain the lack of consensus when defining what an emotion is 8. Nevertheless, two basic orientations can be highlighted. One is the universalistic approach that goes back to Darwin's research on emotional expression and emphasizes the universal character of some basic human emotions, such as fear, anger, happiness, sadness, or disgust 9. Within this broad paradigm, most researchers see emotions as adaptive mechanisms organizing human behavior in ways appropriate to environmental demands 10. In Levenson's words 11:

The prototypical context for human emotions is those situations in which a multi-system response must be organized quickly, where time is not available for the lengthy processes of deliberation, reformulation, planning and rehearsal; where a fine degree of coordination is required among systems as disparate as the muscles of the face and the organs of the viscera; and where adaptive behaviors that normally reside near the bottom of behavioral hierarchies must be

\footnotetext{
${ }^{3}$ For the purpose of this paper, we use ODR in a broad and flexible sense to encompass not only disputes that originate from online transactions, but also off-line disputes handled online. Similarly, the "online" component may be extended to include the use of electronic applications such as video-conferencing, mobile telephony, voIP, etc.
} 
instantaneously shifted to the top.

The second paradigm is normally referred as the "social constructivist" approach 12 , which focuses on emotions as culture-bound artifacts 13. Without denying the hypothesis of universal basic human emotions, social constructivists aim at offering "symbolic", "interpretive" or "intentional" theories of emotions 14. Moreover, some of them have contested the Western oriented character of concepts, expressions and languages dealing with emotions, indicating that "there are no emotional terms which can be matched neatly across language and culture boundaries" 15 .

Beyond these basic differences, the vast majority of researchers would share -at least to some degree- the hypothesis according to which emotions serve some kind of function 16. However, both the notion of "function" itself and the scope of functional explanations broadly differ. Thus, from an evolutionary perspective, the functions of emotions are associated with "recruiting physiological changes", "action readiness", "changing cognitive activity" or "facilitating phylogenetically adaptive responses" 17. As the analysis focuses on the "social functions" of emotions, however, functional explanations are more diffuse:

These converging research traditions indicate that emotions serve social functions. The view is that the consequences of emotions are best examined in light of the recurrent problems in interpersonal and group relations, such as allocating resources fairly, honoring personal contracts, or maintaining friendships (e.g. Averill, 1980; Eibl-Eibesfeldt, 1989; Ekman, 1992; Lutz \& White, 1996). This involves a teleological stance that assumes that emotions can be seen as having functions -not because they were designed, but because they have been selected for on the basis of their adaptiveness, both at the biological level for their contribution to individual fitness, and at the cultural level for their contribution to individual and group functioning To say that emotions resulted from biological and cultural evolution does not mean, however, that they serve an actor well all or most the time they occur 18.

A third approach to the functions of emotions seems closer to the mathematical use of the notion. Thus, without abandoning an adaptive perspective, it suggests relations among specific behaviors rather than teleological causes of them. From this perspective, researchers have emphasized the communicative and relational character of emotions, focusing on their role in signaling social behaviors 19. In their account on basic emotions, Johnson-Laird \& Oatley -following a cognitive approach that goes back to Simon 20- refer to them as "signals" that "have no prepositional content or syntactic structure" and fulfill a "control function" rather than an "informational one" 21. This "control function" consists of redistributing cognitive resources and goals:

[Emotions] arise particularly when individuals have many concurrent goals, including mutually incompatible ones, and their resources of time, ability, and processing power, are too limited to make a fully rational choice. Moreover, social mammals often cannot achieve their more valuable objectives alone, and so they need to interact with others. Co-operation calls for mutual plans, but it is impossible to guarantee that copies of the plan kept by each partner are identical. Competition calls for antagonistic plans, and it is impossible to determine their outcome. The biological system of emotions offers a solution to these problems, 
particularly those that arise from the limits of rational principles to govern or to predict complex social interactions. Emotions enable social species to coordinate their behavior, to respond to emergencies, to prioritize goals, to prepare for appropriate actions, and to make progress toward goals 22 .

The issue that may be raised at this point is whether the functional aspects of emotions are preserved in ODR environments. Most research on ODR and emotions implicitly accepts that ODR techniques (ranging from blind bidding to e-mediation supported by a human mediator) allow participants to be flexible, to adapt their responses to a changing environment, to prioritize their goals and even to properly identify emotions in the opposing party 23. In this view, the beneficial functions of emotions find their place in ODR. But there is also countervailing research supporting the view that ODR may be more successful than traditional ADR in inhibiting or filtering out emotions, especially when using text based techniques 24. In that case, emotions are brought into play as involving negative functions (i.e. signaling hostile behaviors, threatening the other party, inhibiting trust formation, etc.) that make resolution less likely. The ultimate question would be whether the expression of emotions is always beneficial or not and, related to that, whether ODR techniques are able to deal with the functional aspects of emotions at convenience. In our view, the present stage of research both in the field of emotions and ODR makes it difficult to provide something more than reductionist or simplistic answers. It is therefore necessary to look at smaller and more manageable emotional components to assess the pros and cons of ODR in dealing with emotions, as compared to traditional ADR.

\section{Is ODR emotionally limited?}

Since ODR services are currently offering different tools to participants, we propose to distinguish them using the most usual categories in both research and practitioners literature. The following table provides a basic classification of some ADR and ODR tools and techniques, based on different modes of communication. Even though ADR and ODR cannot be simply encapsulated in those main modes of communication (F2FC and CMC, respectively) much comparisons between ADR and ODR deal with advantages and disadvantages of the two modes of communication. The second distinction is to be made between synchronous and asynchronous modes of communication. While synchronous communication refers to real time interactions (individuals interact at the same time in the same physical or virtual place) asynchronous communication does not occur in the same unit of time and participants are involved in the process at different moments.

ADR and ODR tools and techniques, based on different modes of communication

\begin{tabular}{|l|l|l|}
\hline & Face-to-face communication (F2FC) & Computer-mediated communication (CMC) \\
\hline $\begin{array}{l}\text { Synchronous } \\
\text { communication }\end{array}$ & $\begin{array}{l}\text { Negotiation/Mediation } \\
\text { session in a physical place }\end{array}$ & $\begin{array}{l}\text { Instant messaging } \\
\text { Chat } \\
\text { Videoconference } \\
\text { VoiceIP }\end{array}$ \\
\hline $\begin{array}{l}\text { Asynchronous } \\
\text { communication }\end{array}$ & $\begin{array}{l}\text { Shuttle mediation } \\
\text { Caucusing }\end{array}$ & $\begin{array}{l}\text { E-mail } \\
\text { Posting } \\
\text { Online caucusing }\end{array}$ \\
\hline
\end{tabular}


If we consider ODR as a communicative process involving individuals engaged in interactive decision-making, we will need to admit that emotions are an essential component of the individuals' attitudes towards the disputing process, regardless of the specific tool used. Nevertheless, most concerns tend to concentrate on the drawbacks that computer-mediated-communication and online processes present as compared to ADR face-to-face sessions:

The most frequently heard concern about ODR has been that online processes and interactions cannot match the richness of the face-to-face sessions that are at the heart of offline mediation. Face-to-face sessions enable a mediator to regulate who says and hears what simply by physically including or excluding parties from the room. In addition, the mediator gets feedback from the parties both by hearing what is said and by seeing how it is said. Other elements of the mediation process, such as building trust and maintaining a non-hostile environment, are also assisted by behavioral interactions." 25

First, skeptics of ODR question its efficacy in dealing with emotions since online communications, when compared to face-to-face communication, are seen as impersonal, lacking human interaction and unable to express non-verbal cues such as the variable tone, pitch and volume of the participants 26. Accordingly, these missing elements of the interaction increase the risk of miscommunication, inhibiting the development of positive interpersonal relations 27.

Secondly, criticism of ODR as a proper environment for interpersonal relations casts doubts on technical issues that may block the development of trust among parties, such as inadequate confidentiality, security, identity or authenticity 28 . Another criticism that affects trust building is related to the so called "digital divide". In this perspective, ODR does not grant sufficient accessibility to those who are not knowledgeable with information technologies and computers, since it requires a certain level of user sophistication 29. Therefore, it is likely that those who are in a disadvantageous position with respect to technology will tend to withdrawn from ODR services.

Third, it is also believed that the inner constraints of online communications will necessarily circumscribe ODR to a limited range of disputes 30 . Thus, ODR would be an appropriate forum for commercial disputes in which the economic transaction remains the main issue, such as consumers and insurances disputes. But the more complex and multifaceted a dispute becomes, the less suited for ODR techniques.

Finally, some authors have also cautioned that disadvantages of online processes, as compared to face-to-face communication, can lead to insufficient control of the mediator over the negotiating parties 31, imprecise evaluation of the flexibility, strength, feelings or confidence of the other party 32, escalation of negative emotion and, ultimately, negotiation impasse 33 .

Do these different criticisms mean that online negotiations are unable to facilitate the emotional atmosphere to craft successful agreements? Let us consider alternative research in more detail. 
Despite being generally accepted that ODR cannot replicate the setting of a face-to-face interaction, recent findings may moderate some concerns about ODR as an impersonal environment where emotions cannot be used as contextual or interaction cues. In this regard, different studies show that participants in ODR processes do not necessarily feel unwise in the expression of emotions. Rather, they are conveyed through different means. Consider for example the following example by Raines, in which capital letters become "online shouting":

\section{JUST WANT TO BE DONE WITH HER AND NEVER DEAL WITH HER AGAIN! LET'S JUST STOP ALL THE HASSLE AND RETURN MY MONEY! MANY, MANY THANKS! 34}

Van Kleef et al. have shown that in the course of computer-mediated negotiation emotions such as anger, happiness, disappointment, will, and regret have interpersonal effects on negotiators. In this way, "negotiators monitor the opponent's emotions, use those emotions to estimate the opponent's limits, and modify their demands according to the presumed location of those limits 35. Another empirical study by Hammond concludes that ODR "allows disputants to be more thoughtful in their submissions, evaluate their emotions and express them rationally, and engage at their own pace - at all the time when they feel calmer and better able to focus on the issues" 36 . Ben-Ze'ev has recently coined the notion of "detached attachment" to highlight that "the relative anonymity of cyberspace and the ability to only reveal matters we would like to reveal provide an opportunity to guard privacy while increasing emotional closeness and openness" 37.

As regards technological impediments for ODR to build trust among parties or provide incentives for cooperative behavior, Fehr \& Gächter have found that in online situations in which people will never meet again and have no incentives to cooperate, they develop cooperative attitudes such as altruistic punishment. Altruistic punishment of defectors implies that individuals punish other participants even in non repeated situations, although the punishment is costly for them and yields no material gain. For these researchers, "negative emotions towards defectors are the proximate mechanism behind altruistic punishment" 38. In addition, the concerns about ODR deepening the digital divide have also been contested by research showing that the ODR environment may actually work better in disputes where there is a power imbalance 39 .

Concerning the range of disputes that ODR may cover, recent data show its expansion out of the e-commerce domain. Successful ODR providers such as SquareTrade, which has already handled over one and a half-million disputes and has become the world's largest dispute resolution provider 40, now include disputes that arise in the off-line world 41. Currently, there are online negotiation support systems being used in family cases, enterprise bargaining and international disputes 42. Apart from the fact that in some cases, "ODR could be the only feasible dispute resolution system available" 43 and computer-mediated-communication is often the means by which people make first contact with one another 44, the incorporation of new technologies with high penetration in different world areas (i.e. mobile telephony or community radio) may facilitate the development of ODR services beyond its e-commerce origins. 
Finally, as regards insufficient control of the mediator over the parties, research has shown that the asynchronous nature of many online applications may provide practitioners with new tools, such as pre-communication reframing (where messages are previously directed to the mediator, thus enabling him to coach the parties with respect to the further framing of their communication and potentially prevent destructive statements reaching the other party) 45 . In this line, Raines concludes that "reframing is probably easier in an online environment, since the mediator can take the time necessary to compose an appropriate response to an inflammatory statement from a party. A poker face is not required for ODR, as often is for traditional mediation" 46. Even well-know techniques of ODR, such as caucusing with negotiating parties, are given new potentialities in ODR, since "dispute resolution practitioners do not need to concern themselves with party reactions to the amount of time they spend separately with each party." 47.

\section{Recent findings and current applications}

ODR and computer-mediated-communication in general have triggered further research based on experiments and models that compare interactions of individuals and emotional states in both face-to-face interaction and computer-mediatedcommunication. Experiments also include testing the distinct features of synchronous versus asynchronous communication in virtual environments. Although this empirical research is developing only very recently, some interesting findings can be highlighted.

First, as regards access to online environments, the environmental psychology approach has also suggested that both information and emotions play a role in the decisions of users to approach or avoid an environment 48. Thus, environments with a large amount of information are more likely to elicit unpleasant emotions, such as the user feeling that he or she has lost control over interaction with the environment. The emotion-approach hypothesis predicts that users will want to approach pleasant, stimulating, and controllable virtual environments 49. In the area of e-commerce findings suggest that information load and emotions influence virtual exploratory and shopping decisions 50.

Second, research indicates that the specific mode of communication has an effect on emotions. But here results are contradictory. On the one hand, Pesendorfer \& Koeszegi state that, "synchronous negotiation mode leads to less friendly, more affective and more competitive negotiation behavior. Asynchronous communication mode leads to more exchange of private and task-oriented information and to a more friendly communication style. These results suggest that de-individuation and escalating effects might be caused by communication mode rather than by the ability of the media to transmit social cues" 51 . On the other, empirical evidence from a study of ninety-eight mediators concludes that synchronous on-line communication (on-line chat) had a much higher rate of win-win solutions compared to delayed communication via e-mail 52 . Another study comparing e-mail communication versus face-to-face communication also finds that the latter may contain more positive emotional communication than email communication, and "using F2FC before or after e-mail communication may lead to more accurate perception of the other" 53. In a similar vein, Nadler compared in an online negotiation simulation negotiators who were allowed to engage in telephone "small talk" and negotiators who were not: "Small Talk' negotiators were over four times as likely to reach an agreement as 'No Small Talk' negotiators. In the negotiation 
simulation involved in this experiment, a seemingly trivial intervention-a preliminary, brief, and informal chat on the telephone-increased the likelihood that the e-mail negotiations that followed would be characterized by cooperation, information exchange, reciprocity, liking, trust, and ultimately, agreement. These negotiators had the opportunity to establish common ground with the other negotiator through small talk, even if the basis for common ground was exceedingly trivial" 54. Finally, it has also been stated that using both CMC and FTF for discussion enhances job satisfaction more than using just one media 55.

Third, recent prototypes try to empirically apply emotions in computer-mediatedcommunication. For instance, Holzman and Pottenger use a linguistic model to tag chat conversation with emotion tags and thus discriminate emotional from non-emotional content 56. Tatai et al. have developed a multimodal Internet chatterbot system with an emphasis on displaying and transmitting emotions between the chat partners. They found that in its 24 emotions model, "emotion icons such as terror, loathing, fear, rage and grief were used by only 3 percent of all chatters and made up only 1 percent of all emotions being used, whereas chatters reported missing certain composite emotions, such as the "winky" state ";-)". Researchers concluded that "chat requires a special set of emotions that differs from emotions used in everyday life" 57. Boucouvalas has examined alternative non-video based means to achieve expressive Internet communications. His model applies tagging and parsing techniques to extract emotional states from the content of typed-text sentences 58. Finally, Ohene-Djan have developed an information visualization interface that enables a user to input a real-time continuous flow of their predominant emotion incorporating degrees of uncertainty relative to other choices. Such a color spectrum provides an insight into when, how and with what degree of certainty opinions were developed and changed over time 59.

Ultimately, what these models have in common is the idea that emotions emerge in online interactions following specific patterns that can be identified, retrieved and analyzed with a variety of technologies. In this way, they all tend to preserve emotions as "social functions", "contextual cues" or "indexes" in virtual environments. While ODR services may certainly benefit from this specific research on emotions applied to specific modes of communication, it is also necessary to be cautious about its results, since further empirical studies, tests, and models are required to contrast the validity of them in a more general level.

\section{Conclusion}

In this paper we have reviewed recent literature on the expression of emotions in ODR, including the criticisms and advantages attributed to ODR as compared to off-line techniques. It seems clear at this stage of research that emotions emerge in online environments as properties of the interaction, shaping individual attitudes towards the communicative and informational flow. Results obtained so far suggest than, contrary to traditional views, ODR cannot be considered an inferior medium for the transmission of emotions, as compared with offline ADR. Rather, emotions are expressed in a different way as they emerge in off-line, face-to-face environments. In this line, ODR experts suggest that online communication culture has developed its own paralinguistic cues to express emotions (i.e. through special characters, emoticons, use of capital letters, etc.). 
Even though the particular display of emotions needs more empirical research in different areas to be fully understood, the study of emotions may have a lot more to do with online processes than has hitherto been supposed. As the number of technologies available to ODR is expanding, we may anticipate the parallel development of a specific culture of emotional expression. This also offers a promising land for research in ODR.

\section{References}

1 See, for example, B L Fredrikson, 'The Value of Positive Emotions', American Scientist, Vol. 91, 2003, 330-336; R Lane and L Nadel (eds) Cognitive Neuroscience of Emotion, New York, Oxford University Press, 2000; A L Hinton (ed) Biocultural Approaches to the Emotions, Cambridge (UK), Cambridge University Press, 1999; S Planalp, Communicating Emotion: Social, Moral, and Cultural Processes, New York: Cambridge University Press, 1999. P Ekman, and R J Davidson (eds) The Nature of Emotion: Fundamental Questions, New York: Oxford University Press, 1994; C Lutz and G M White, 'The Anthropology of Emotions', Annual Review of Anthropology, Vol. 15, 1986, 405-36.

2 C Castelfranchi F Giardini and F Marzo, "Relationships between rational decisions, human motives, and emotions', Mind \& Society, Vol. 5, 2006, 173-197.

3 R J Dolan, 'Emotion, Cognition, and Behavior', Science, Vo. 298, 2002, 1191-1194; M L Käsermann et al, 'The Study of Emotional Processes in Communication: I. Measuring Emotionalization in Everyday Face-to-Face Communicative Interaction', Behavior Research Methods, Instruments \& Computers, Vol. 32 (1), 2000, 33-46.

4 Op cit, note 1.

5 P Johnson-Laird and K Oatley, 'Basic Emotions, Rationality, and Folk Theory', Cognition and Emotion, Vol. 6, 1992, 201-223.

6 Op cit, note 5. See also K Oatley and P Johnson-Laird, 'Towards a Cognitive Theory of Emotion', Cognition \& Emotion, Vol. 1, 1987, 51-58; C M Worthman, 'Emotions: You Can Feel the Difference' in A. L. Hinton (ed.) Biocultural Approaches to the Emotions, Cambridge (UK): Cambridge University Press, 1999, 41-74.

7 M E Lund 'A Focus on Emotion in Mediation Training', Family \& Conciliation Courts Review, Vol. 38 (1), 2000, 62-68.

8 R J Davidson, 'On Emotion, Mood, and Related Affective Constructs' in P Ekman \& R J Davidson, op cit note 1, 51-55.

9 C Darwin, The Expression of the Emotions in Man and Animals. London: John Murray, 1872; P Ekman, 'Universals and Cultural Differences in Facial Expressions of Emotions' in J Cole (ed) Nebraska Symposium on Motivation, Vol. 19, Lincoln: University of Nebraska Press, 1972, 207-283; P Ekman, 'Facial expression and emotion', American Psychologist, Vol. 48, 1993: 384-392; R S Lazarus, 'Universal Antecedents of Emotions' in P Ekman and R J Davidson, op cit note 1: 163-171; J Tooby, and L Cosmides, 'The Past Explains the Present: Emotional Adaptations and the Structure of Ancestral Environments', Ethology and Sociobiology, Vol. 11, 1999: 37542; A Damasio, The Feeling of What Happens: Body and Emotion in the Making of Consciousness. New York, San Diego, London: Harcourt Brace \& Company, 1999; A Damasio, 'A Second Chance for Emotion' in R Lane and R L Nadel (eds) Cognitive Neuroscience of Emotion, New York: Oxford University Press, 2000, 12-23; B Kolb and L Taylor, 'Facial Expression, Emotion, and Hemispheric Organization' in R Lane, 
and L Nadel (eds), Cognitive Neuroscience of Emotion, New York: Oxford University Press, 2000, 62-83.

10 R W Levenson, 'Emotion and the Autonomic Nervous System: A Prospectus for Research on Autonomic Specificity' in H Wagner (ed) Social Psychophysiology and Emotion: Perspectives on theory and clinical applications, London: Wiley, 1984: 1742; R W Levenson, 'The Search for Autonomic Specificity' in P Ekman and R J Davidson, op cit note 1, 252-257; R S Lazarus, Emotion and Adaptation, New York: Oxford University Press, 1991; K R Scherer, "Emotion Serves to Decouple Stimulus and Response," in P Ekman and R J Davidson, op cit note 1, 127-136.

11 Levenson, op cit note 10.

12 See, for example, D Keltner and J J Gross, 'Functional Accounts of Emotions', Cognition and Emotion, Vol. 13 (5), 1999, 467-480.

$13 \mathrm{~J}$ Averill, 'A Constructivist View of Emotion' in R Plutchik and H Kellerman (eds) Theories of Emotion, New York: Academic Press, 1980, 305-340; A R Hochshild, The Managed Heart: Commercialization of Human Feeling. Berkeley: University of California Press, 1983; C Geertz, 'From the Native's Point of View: On the Nature of Anthropological Understanding' in R A Shweder and R A LeVine (eds) Culture Theory: Essays on Mind, Self, and Emotion. Cambridge: Cambridge University Press, 1994, 123-136; H Flam, 'Emotional 'Man': I. The Emotional Man and the Problem of Collective Action', International Sociology, Vol. 5 (1), 1990, 39-56; H Flam, 'Emotional 'Man': II. 'Corporate Actors as Emotion-Motivated Emotion Managers', International Sociology, Vol. 5 (2), 1990, 225-234; C Lutz and G White, 'The Antropology of Emotions', Annual Review of Anthropology, Vol. 15, 1986: 405-436; A Wierzbicka, 'Talking about Emotions: Semantics, Culture, and Cognition', Cognition and Emotion, Vol. 6, 1992, 285-319; A Wierzbicka, 'Emotion, Language, and Cultural Scripts' in S Kitayama and H R Markus (eds) Emotion and Culture: Empirical Studies on Mutual Influence, Washington, DC: American Psychological Association, 1994, 133-196; M W Morris and D Keltner, 'How Emotions Work: An Analysis of the Social Functions of Emotional Expression in Negotiation', Research Paper No. 1591. Graduate School of Business, Stanford University, 1999.

14 R Shweder, 'You're not Sick, you're Just in Love: Emotion as an Interpretive system' in P Ekman and R J Davidson, op cit note 1.

15 Lutz and White, op cit note 13; Wierzbicka, op cit note 13.

16 E T Rolls, The Brain and Emotion. Oxford: Oxford University Press, 1999; Keltner and Gross, op cit note 12; D Keltner and J Haidt, 'Social Functions of Emotions at Four Levels of Analysis', Cognition and Emotion, Vol. 13 (5), 1999, 505-521.

17 P Ekman and R J Davidson, op cit note 1.

18 Keltner and Haidt, op cit note 16;

19 Oatley and Johnson-Laird, op cit note 5; Lazarus, op cit note 10; N Fridja, 'Emotions are Functional, Most of the Time', in P Ekman and R J Davidson, op cit note 1, 112-122; Morris and Keltner, op cit note 13.

$20 \mathrm{H}$ A Simon, 'Motivational and Emotional Controls of Cognition' Psychological Review, Vol. 74, 1967, 29-39.

21 Johnson-Laird and Oatley, op cit note 5; see also I Wright, A Sloman \& L Beaudoin, 'Towards a Design-Based Analysis of Emotional Episodes', Philosophy, Psychiatry \& Psychology, Vol. 3 (2), 1996, 101-126.

22 Johnson-Laird and Oatley, op cit note 5.

23 A M Braeutigam, 'Fusses that Fit Online: Online Mediation in non-commercial Contexts', Appalachian Journal of Law, Vol. 5, 2006, 275-301.

24 See B Zondag and A Lodder in this volume. 
25 E Katsch, 'Bringing Online Dispute Resolution to Virtual Worlds: Creating Processes through Code', New York Law School Law Review, Vol. 49 (1), 2005, 271291.

26 A Shah, 'Using ADR to Resolve Online Disputes', Richmond Journal of Law and Technology, Vol. 10, 2004, 25-56; J Nadler, 'Rapport in Legal Negotiation: How Small Talk Can Facilitate E-Mail Dealmaking', Harvard Negotiation Law Review, Vol. 9, 2004, 223-251; J W Goodman, 'The Pros and Cons of Online Dispute Resolution: An Assessment of Cyber-Mediation Websites', Duke Law \& Technology Review, Vol. 4, 2003, 1-31.

27 Shah, ibid.; Nadler, ibid.; Goodman, ibid.; R A Friedman and S C Currall, E-Mail Escalation: Dispute Exacerbating Elements of Electronic Communication' Nashvill: Vanderbilt University, 2004; R Birke and L E Teitz, 'U.S. Mediation in 2001: The Path that Brought America to Uniform Laws and Mediation in Cyberspace' American Journal of Comparative Law, Vol. 50, 2002, 181-213; E Katsch, E Rifkin \& A Gaitenby, 'E-Commerce, E-Disputes, and E-Dispute Resolution: In the Shadow of “eBay Law', Ohio State Journal on Dispute Resolution, Vol. 15, 2000, 705-714; J B Eisen, 'Are We Ready for Mediation in Cyberspace?', BYU Law Review, 1998, 1305.

28 Shah, ibid.; Nadler, ibid.; Birke \& Teitz, ibid.; E Katsh, 'Dispute Resolution in Cyberspace', Connecticut Law Review, Vol. 58, 1996, 953-971.

29 Shah, ibid.; Birke and Teitz, ibid.; Eisen, ibid.

30 Shah, ibid.; Goodman, ibid.

31 Katsh, Rifkin and Gaitenby, op cit note 27.

32 Nadler, op cit note 26.

33 Eisen, op cit note 27.

34 S S Raines, 'Can Online Mediation Be Transformative? Tales from the Front', Conflict Resolution Quarterly, Vol. 22 (4), 2005, 437-451.

35 G Van Kleef, C K W de Dreu, A S R Manstead, 'The Interpersonal Effects of Emotions in Negotiations: A Motivated Information Processing Approach', Journal of Personality and Social Psychology, Vol. 87 (4) 2004, 510-528; G Van Kleef, C K W de Dreu, D Pietroni, A S R Manstead, 'Power and emotion in negotiation: Power moderates the interpersonal effects of anger and happiness on concession making', European Journal of Social Psychology, Vol. 36, 2006, 557-581; G Van Kleef, C K W de Dreu, A S R Manstead, 'Supplication and Appeasement in Conflict and Negotiation: The Interpersonal Effects of Disappointment, Worry, Guilt, and Regret', Journal of Personality and Social Psychology, Vol. 91 (1), 2006, 124-142.

36 A G Hammond, "How Do You Write "Yes"?: A Study on the Effectiveness of Online Dispute Resolution', Conflict Resolution Quarterly, Vol. 20 (3), 2003, 261-86.

37 A Ben-Ze'ev, 'Privacy, Emotional Closeness, and Openness in Cyberspace', Computers in Human Behavior, Vol. 19, 200, 451-467. See also A Ben-Ze'ev, Love Online: Emotions on the Internet, Cambridge: Cambridge University Press, 2004.

38 E Fehr, and S Gächter, 'Altruistic Punishment in Humans', Nature, Vol. 415, 2002, 137-140.

39 Braeutigam, op cit note 23; Hammond, op cit note 36; N Alexander, 'Mobile Mediation: How Technology is Driving the Globalization of ADR' Hamline Journal of Public Law and Policy, Vol. 27, 2006: 243-262.

40 Katsch, op cit note 25

41 Braeutigam, op cit note 39

$42 \mathrm{~J}$ Zeleznikow and E Bellucci, 'Family Winner: Integrating Game Theory and Heuristics to Provide Negotiation Support' (http://www.jurix.nl/pdf/j03-03.pdf ).

43 Katsch, op cit note 25. 
$44 \mathrm{Y}$ Kato, and $\mathrm{K}$ Akahori, 'E-mail Communication Versus Face-to-Face Communication: Perception of Other's Personality and Emotional State', 2003 (http://www.ak.cradle.titech.ac.jp/papers/pdf/yuukiEDMEDIA2004.pdf).

45 Alexander, op cit note 39.

46 Raines, op cit note 34.

47 Alexander, op cit note 39.

48 H M Huang, 'Modeling Virtual Exploratory and Shopping Dynamics: An Environmental Psychology Approach', Information \& Management, Vol. 41, 2003, 3949 Ibid.

$50 \mathrm{Ibid}$.

51 E M Pesendorfer and S T Koeszegi, 'The Effect of Communication Mode in Enegotiations',

2005

[http://nebel.site.uottawa.ca/workshop/Papers/pesendorfer_koeszegi.pdf].

52 J Tan, D Bretherton, G Kennedy, 'Negotiating Online", 2004 [http://www.odr.info/unforum2004/tan.htm].

53 Kato and Akahori, op cit note 44.

54 Nadler, op cit note 26.

$55 \mathrm{~T}$ Mukahi, M Nakamura, G Corbitt 'The impacts of Computer-Mediated Communication and Face-to-Face Communication in actual organizations', 2003 [http://www.pacis-net.org/file/2003/papers/poster/103.pdf].

56 L E Holzman and W M Pottenger 'Classification of Emotions in Internet Chat: An Application of Machine Learning Using Speech Phonemes,' Technical Report, 2003 [http://www3.lehigh.edu/images/userImages/cdh3/Page_3456/LU-CSE-03-002.pdf].

57 G Tatai, L Laufer, A Szaló, A Csordás, 'On Transmitting Emotions - Experiments on Multimodal Presence over the Internet' 6th International Workshop on Presence, 2004 [http://www.presence-research.org/p2003.html].

58 A Boucovalas, 'Real Time Text-to-Emotion Engine for Expressive Internet Communications' in G Riva, F Davide, W A IJsselsteijn (eds) Being There: Concepts, Effects and Measurement of User Presence in Synthetic Environments, Amsterdam, Ios Press, 2003, 306-318.

59 J Ohene-Djan, A Sammon, R Shipsey 'Colour Spectrum's of Opinion: An Information Visualisation Interface for Representing Degrees of Emotion in Real Time', Proceedings of the Information Visualization (IV'06), p 80, 2006. 